\begin{tabular}{|c|l|}
\hline Title & Near infrared study of water-benzene mixtures at high temperatures and pressures \\
\hline Author(s) & Jin, Y usuke; Ikawa, Shun-ichi \\
\hline Citation & $\begin{array}{l}\text { The Journal of Chemical Physics, 121(6), 2694 2700 } \\
\text { https://doi.org/10.1063/1.1769356 }\end{array}$ \\
\hline Issue Date & 2004 \\
\hline Doc URL & http://hdl.handle.net/2115/1395 \\
\hline Rights & Copyright $\odot 2004$ A merican Institute of Physics \\
\hline Type & article \\
\hline File Information & JCP121-6.pdf \\
\hline
\end{tabular}

Instructions for use 


\title{
Near infrared study of water-benzene mixtures at high temperatures and pressures
}

\author{
Yusuke Jin and Shun-ichi Ikawa \\ Division of Chemistry, Graduate School of Science, Hokkaido University, Sapporo, 060-0810, Japan
}

(Received 3 March 2004; accepted 14 May 2004)

\begin{abstract}
Near-infrared absorption of water-benzene mixtures has been measured at temperatures and pressures in the ranges of 473-673 K and 100-400 bar, respectively. Concentrations of water and benzene in the water-rich phase of the mixtures were obtained from the integrated absorption intensities of the $\mathrm{OH}$ stretching overtone transition of water and the $\mathrm{CH}$ stretching overtone transition of benzene, respectively. Using these concentrations, the densities of the water-rich phase were estimated and compared with the average densities before mixing, which were calculated from literature densities of neat water and neat benzene. It is found that anomalously large volume expansion on the mixing occurs in the region enclosed by an extended line of the three-phase equilibrium curve and the one-phase critical curve of the mixtures, and the gas-liquid equilibrium curve of water. Furthermore, magnitude of the relative volume change increases with decreasing molar fraction of benzene in the present experimental range. It is suggested that dissolving a small amount of benzene in water induces a change in the fluid density from a liquidlike condition to a gaslike condition in the vicinity of the critical region. (C) 2004 American Institute of Physics.
\end{abstract}

[DOI: $10.1063 / 1.1769356]$

\section{INTRODUCTION}

Water at high temperatures and pressures in the subcritical to supercritical region has been paid much attention these days, due to its intriguing properties and possibility of various applications. Differing from ambient water, the high temperature water is a good solvent for nonpolar compounds such as hydrocarbons, ${ }^{1-5}$ which are usually regarded as hydrophobic substances. For example, water and benzene become completely miscible at any mixing ratio at temperatures and pressures above $573 \mathrm{~K}$ and 200 bar, respectively, forming a homogeneous phase. This situation is clearly understood by a pressure-temperature phase diagram of the mixture in Fig. 1, in which open circles indicate typical experimental points in the present study. Such waterhydrocarbon mixtures are important in a wide range of industries including oil refineries, petrochemical industry, coal gasification and liquefaction, and a number of thermodynamical data have been reported so far. ${ }^{8-13}$ These data are also useful for designing an environment-protecting technology where hydrothermal reactions are applied to destruction of toxic waste chemicals. ${ }^{14-18}$ In contrast to those extensive studies on the thermodynamic properties, molecular-level structures of the high-temperature mixtures had remained largely unknown until quite recently.

In the recent studies, ${ }^{19,20}$ we have shown that in situ infrared measurements are useful for obtaining molecularlevel information of the high-temperature mixtures. We have found that water concentration in aromatic hydrocarbons increases rapidly with increasing temperature, and the relative ratio of hydrogen-bonded dimeric water to monomeric water increases with temperature up to $523 \mathrm{~K}$ at a constant pressure of 100 bar. This fact indicates that the effect of the solubility increase on the monomer-dimer equilibrium is superior to the entropy effect that is unfavorable to water-water association. At higher temperatures, the component bands attributed to the monomers and the dimers merged into a single band and could not be resolved meaningfully. This is attributed to rapid exchange between the hydrogen-bonded and hydrogenbond-free states of the $\mathrm{OH}$ groups due to temperatureaccelerated rotation of molecules within the water clusters. Moreover, using the concentrations of both water and hydrocarbons, we have found anomalous volumetric behavior of the mixtures in the vicinity of the critical region. For example, volume expansion on the mixing of water and benzene becomes as large as $300 \%$ at $573 \mathrm{~K}$ and 100 bar. $^{21}$ Similar phenomena have been observed for water-toluene and water-ethylbenzene mixtures. ${ }^{22}$ Thus the anomalous volume expansion is considered to be common to the mixing of water and hydrophobic hydrocarbons in the vicinity of the critical region. These findings have been obtained for the first time by the in situ spectroscopic measurements of one phase, the hydrocarbon-rich phase, of the two coexisting fluid phases. The volumetric concentrations of components and then the density of the one phase in the two-phase coexisting region cannot be obtained by ordinary analytical techniques such as gas chromatography and Karl-Fisher titration.

It is intriguing what volumetric behavior the water-rich phase exhibits in the same temperature-pressure region. To examine this, we require obtaining the concentrations of both water and hydrocarbons in the water-rich phase. However, we were unable to measure the concentrations in the waterrich phase due to the too strong infrared absorption of water. Therefore, we have attempted to measure near-infrared overtone absorption, which is a few orders of magnitude weaker than the fundamental absorption and convenient for quantitative analysis. We have observed the overtone $\mathrm{OH}-$ 


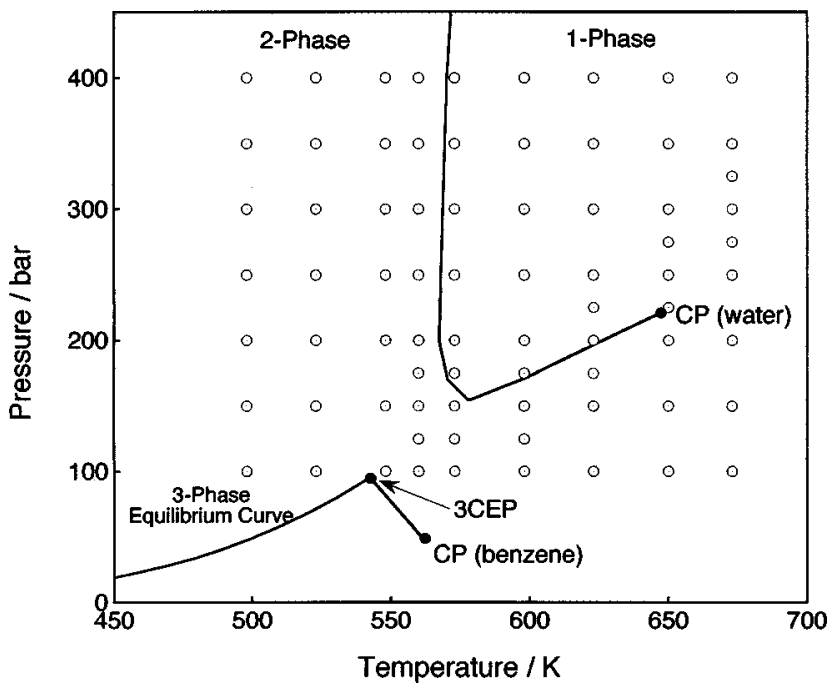

FIG. 1. Pressure-temperature phase diagram for water-benzene mixtures drawn by use of literature data (Refs. 4, 5). CP: critical point (water: 647.14 K, 220.6 ba; benzene: $562.05 \mathrm{~K}, 48.95$ bar) (Ref. 6), 3CEP: three-phase critical end point (542.6 K, 94.6 bar) (Ref. 7). Open circles: typical experimental points in the present study.

stretching absorption of neat water at temperatures in the $373-673 \mathrm{~K}$ range and at pressures in the 20-400 bar range. ${ }^{23}$ From analysis of the molar absorption intensities in the gas and gaslike regions, enthalpy for dimerization has been estimated to be $15 \pm 3 \mathrm{~kJ} / \mathrm{mol}$. At densities in liquid and liquidlike regions, we have found a good correlation between the molar absorption intensities and the first moments of the absorption bands and suggested using the correlation for estimating water concentration in aqueous mixtures. It is worthwhile to mention here that the absorption intensities of infrared and near-infrared bands of water have sometimes been used to characterize the hydrogen bonding state of high-temperature water, ${ }^{24-28}$ but not to estimate a water concentration. The present paper reports near-infrared measurements of the water-rich phase of the water-benzene mixtures. Concentrations of both water and benzene are estimated from respective integrated intensities of near-infrared absorption bands, and the volumetric behavior of the water-rich phase is discussed.

\section{EXPERIMENT}

\section{A. Apparatus and procedure}

The high-pressure cell used has been described previously. ${ }^{23}$ In brief, the cell body is made of Hastelloy (anticorrosion nickel-based superalloy) and the windows are colorless sapphire cylinders. The effective aperture for optical transmission is $6 \mathrm{~mm}$ and the optical path length of a sample is $1.46 \mathrm{~mm}$. The temperature and pressure of the sample fluid are measured with a thermocouple and a pressure transducer of a semiconductor strain gage, respectively. The cell has three sample inlets. The one on the top is for initial filling of sample liquids and the two on the side are for transmitting compressed liquids into the cell with a syringe pump for liquid chromatography. First, the cell was filled with water and benzene and the level of the water-benzene

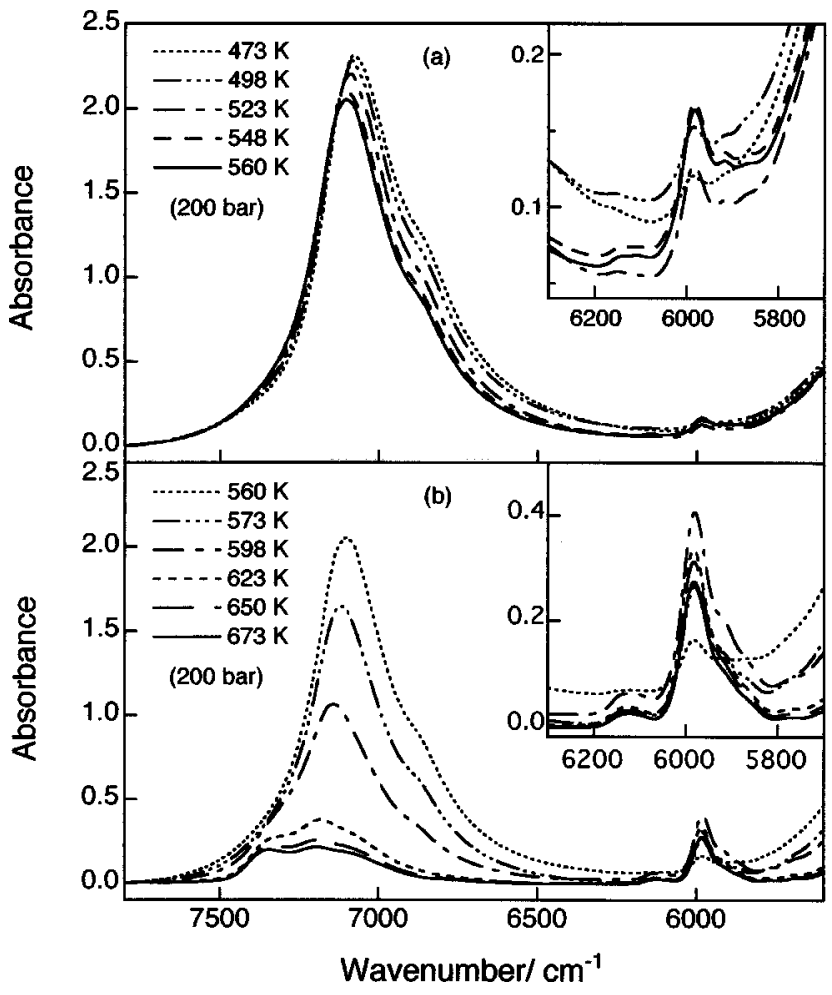

FIG. 2. Near-infrared absorption spectra of the water-rich phase of waterbenzene mixtures at temperatures in the ranges of $473-560 \mathrm{~K}$ (a) and 560$673 \mathrm{~K}(\mathrm{~b})$ at a constant pressure of 200 bar.

interface was adjusted so as to be definitely above the optical axis to measure the water-rich phase. The initial volume ratio of water and benzene was 4.5:1.5, which corresponds to 0.063 of a molar fraction of benzene. Then the cell was slowly heated up to an intended temperature. During the heating process, it was necessary to bleed a small amount of the sample from the water phase to avoid overpressure. This was done through the lower one of the two side inlets of the cell mentioned above by fine control of a needle valve, which is connected to a tee-union between the cell and the pressure transducer. Distilled and deionized water with electric conductivity of $0.2 \mu \mathrm{S} \mathrm{cm}^{-1}$ was used, and spectroscopic grade benzene from Nacalai tesque was used as received.

Spectral measurements were performed with a PerkinElmer System 2000 Fourier-transform spectrometer, which was equipped with a near-infrared source and a near-infrared detector. Near-infrared transmission spectra were measured with $2 \mathrm{~cm}^{-1}$ resolution at sample temperatures in the $473-$ $673 \mathrm{~K}( \pm 1 \mathrm{~K})$ range and pressures in the $100-400$ bar $( \pm 0.5$ bar) range.

\section{B. Observed spectra}

Figure 2 displays observed near-infrared spectra of the water-rich phase of the water-benzene mixtures. Bands centered around $7000 \mathrm{~cm}^{-1}$ are assigned to the $\mathrm{OH}$ stretching overtone transition of water and small bands at $6000 \mathrm{~cm}^{-1}$ to the $\mathrm{CH}$ stretching overtone transition of benzene dissolved in the water phase. Inlet figures show benzene bands on an expanded scale. Temperature-dependent change in the absorption is obviously different between the two temperature 


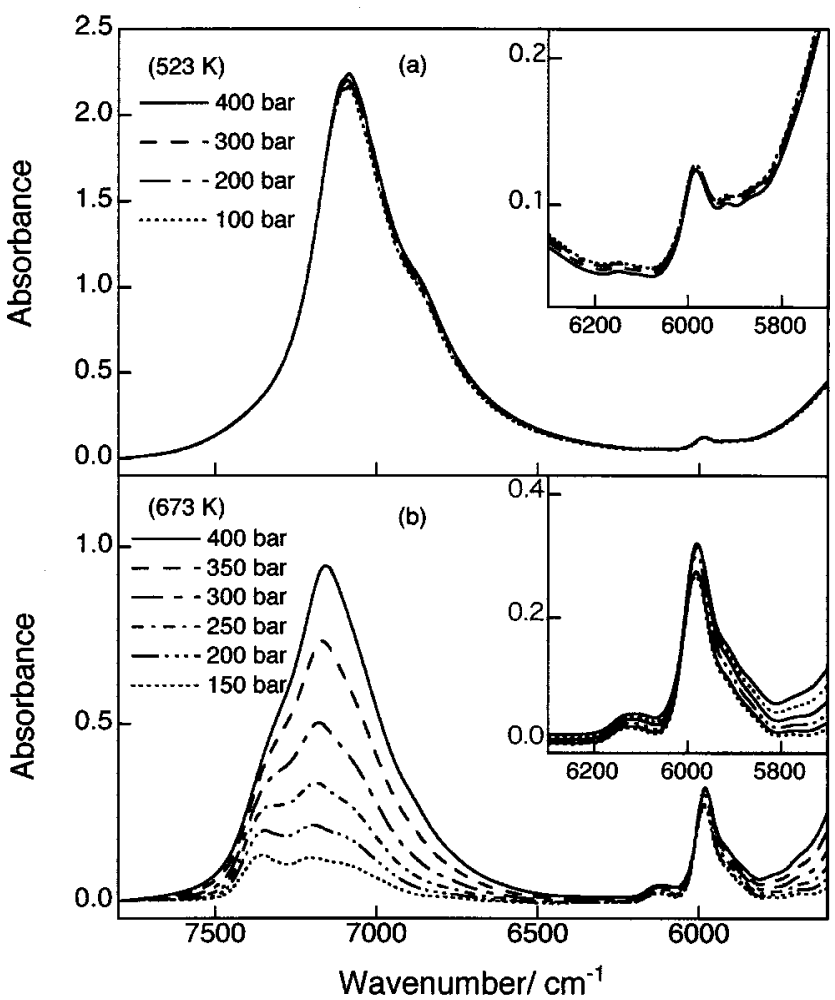

FIG. 3. Near-infrared absorption spectra of the water-rich phase of waterbenzene mixtures at temperatures $523 \mathrm{~K}$ (a) and $673 \mathrm{~K}(\mathrm{~b})$, and at pressures in the range of 100-400 bar.

ranges. The absorption of water slowly decreases with increasing temperature from 473 to $560 \mathrm{~K}$ at a constant pressure of 200 bar, while the absorption of benzene exhibits a relatively large increase as shown in Fig. 2(a). At higher temperatures, on the other hand, the water absorption rapidly decreases with increasing temperature and the benzene absorption steeply increases as the temperature increases from 560 to $573 \mathrm{~K}$ as shown in Fig. 2(b). Figure 3 shows pressure dependence of the absorption. At $523 \mathrm{~K}$ (a), absorption of both water and benzene exhibits little change as the pressure increases from 100 to 400 bar. On the contrary, at $673 \mathrm{~K}$ (b), the water absorption rapidly increases with increasing pressure, while the benzene absorption changes only a little.

These absorption changes reveal how the concentrations vary with temperature and pressure, since the strength of the absorption is approximately proportional to a concentration of the corresponding component, and therefore may be reasonably understood by referring to a phase diagram of the mixtures shown in Fig. 1. The present experimental results indicate that, in the two-phase region, the water concentration decreases a little while the benzene concentration increases with increasing temperature at a constant pressure. Effect of pressure on both the concentrations of water and benzene is very small in the present pressure range in the two-phase region. On the other hand, at higher temperatures in the two-phase region near the critical curve and in the one-phase region, the water concentration increases significantly with increasing pressure, while the benzene concentration moderately changes. For further discussion, values of the concentrations are estimated in the following section.

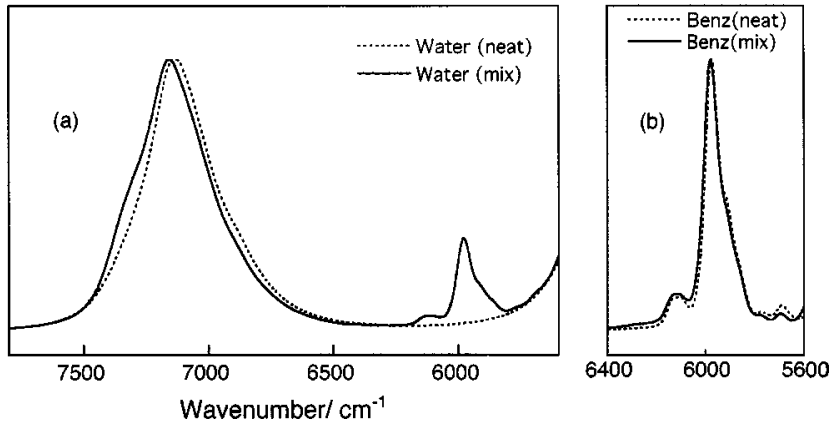

FIG. 4. Comparison of absorption profile of the water-benzene mixture with neat water (a) and neat benzene (b) at $673 \mathrm{~K}$ and 400 bar. The spectra have been normalized at the peak maximum.

\section{DISCUSSION}

\section{A. Estimates of concentrations}

To estimate concentrations from the observed integrated band intensities, we need molar absorption intensities. It is known that the molar absorption intensity of the $\mathrm{OH}-$ stretching transition of water depends on the hydrogenbonding state of the water molecules. Allowing for this, we estimate the molar absorption intensity of water in aqueous mixtures using a correlation between the molar absorption intensity and the first band moment for neat water, which has been obtained in the previous paper. ${ }^{23}$ Since both quantities are mainly determined by the hydrogen-bonding state of water molecules, we have assumed that the correlation can be applied to the aqueous mixtures in which the molar absorption intensity is also determined by the hydrogen-bonding state. The first band moment can be obtained from normalized absorption profile on condition that the absorption does not significantly overlap with other absorption. Then, the molar absorption intensity is given by the correlation shown in Fig. 7 of the previous paper, ${ }^{23}$ which is represented by the following expression:

$$
\begin{aligned}
\left(A^{m} / \mathrm{cm} \mathrm{mol}^{-1}\right)= & 7.41 \times 10^{7}-2.13 \times 10^{4}\left(\bar{\nu} / \mathrm{cm}^{-1}\right) \\
& +1.53\left(\bar{\nu} / \mathrm{cm}^{-1}\right)^{2},
\end{aligned}
$$

where, $A^{m}$ and $\bar{\nu}$ denote the molar absorption intensity and the first band moment, respectively. Then the molar concentration of water is given from the observed intensity $A_{\mathrm{obs}}$ as

$\left(C_{W} / \mathrm{mol} \mathrm{l}^{-1}\right)=10^{3} \times\left(A_{\mathrm{obs}} / \mathrm{cm}^{-2}\right) /\left(A^{m} / \mathrm{cm} \mathrm{mol}^{-1}\right)$.

Uncertainty of the concentration estimates from this method is hard to assess at present, but may be less than $10 \%$.

For benzene, we estimate concentrations by directly comparing the absorption intensities of mixtures and neat benzene, using literature data of concentrations of neat benzene at the same temperature and pressure. ${ }^{29}$ We assume this procedure to be reasonable, taking into account the fact that the absorption profile of benzene changes little on the transfer from the neat liquid to the mixture as shown in Fig. 4. Figure 4(a) compares the absorption bands of the mixture and neat water, both of which are normalized at the peak 


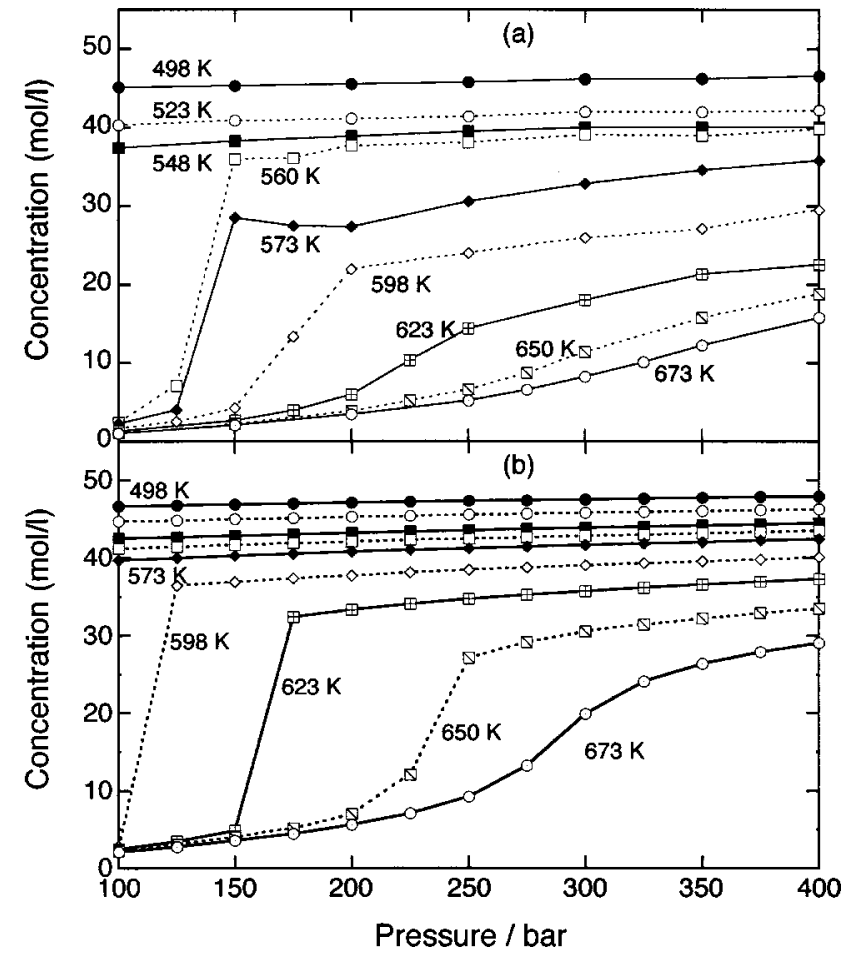

FIG. 5. Plots of concentrations of water in the water-rich phase of the water-benzene mixtures (a) and in neat water (b) against pressure at each of the experimental temperatures.

maximum, and Fig. 4(b) compare the absorption band of neat benzene and that of the mixture obtained by subtracting absorption of water. It is found that the absorption of benzene exhibits only a very small high-frequency shift from neat liquid to the mixture. This fact indicates that the overtone absorption of benzene is insensitive to change in the molecular environment, and it is reasonable to obtain the benzene concentrations in the mixtures from the relative ratio of the absorption intensities. The absorption of water, on the other hand, shows a little larger high-frequency shift, and the bump around $7300 \mathrm{~cm}^{-1}$, which is attributed to a remnant of the $R$ branch of the rotational structure, ${ }^{23}$ increases by dissolution of benzene. These changes in the band profile indicate a decrease in degree of the hydrogen bonding and an increase in proportion of the freely rotating water molecules.

The resulting concentrations of water and the molar fractions of benzene given by

$$
x=\frac{C_{B}}{C_{W}+C_{B}}
$$

are plotted against pressure at each temperature in Figs. 5 and 6. It should be mentioned here that all the values of the water concentrations in the mixtures (a) are smaller than the corresponding values of neat water (b) taken from the literature. ${ }^{30}$ The relative difference is particularly large at points slightly above the vapor pressure curve of water. For example, the concentration in the mixture at $573 \mathrm{~K}$ and 100 bar is an order of magnitude smaller than that of neat water. This is related to an anomalous volumetric behavior of the mixtures discussed in the following section. The molar fractions of benzene in the mixtures show significant tempera-

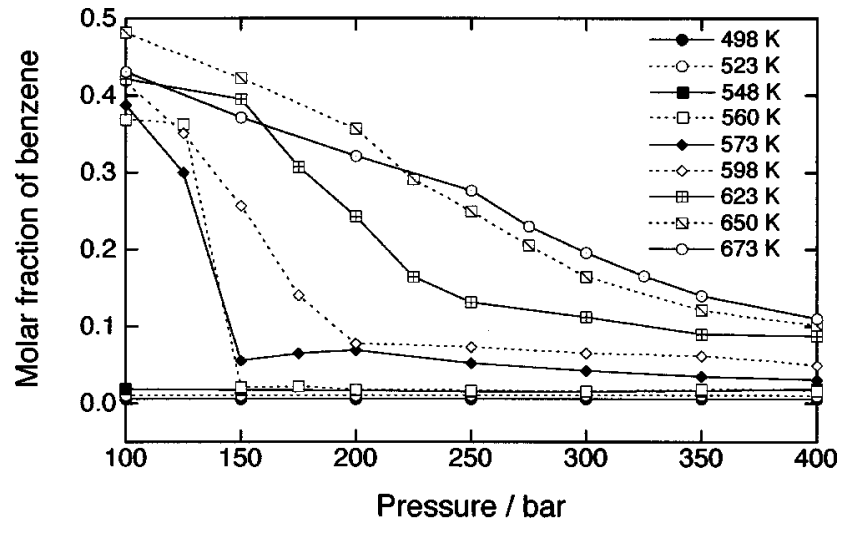

FIG. 6. Plots of molar fraction of benzene in the water-rich phase of the water-benzene mixtures against pressure at each of the experimental temperatures.

ture dependence. At temperatures in the 498-560 $\mathrm{K}$ range, where all the experimental points are in the two-phase region, the molar fractions are very small and approximately independent of pressure except at the two lowest pressures at $560 \mathrm{~K}$. The estimated molar fractions are $2.5 \times 10^{-3}$, $6.0 \times 10^{-3}$, and $1.3 \times 10^{-2}$, at 498,523 , and $548 \mathrm{~K}$, respectively. These values may suffer from large uncertainty due to considerably weak absorption of benzene at lower temperatures and a further study is required to discuss quantitatively. Nevertheless, the present results indicate that the solubility of benzene in water is very small and approximately independent of pressure at temperatures lower than $560 \mathrm{~K}$ in the two-phase region. At higher temperatures, on the other hand, the molar fractions are significantly large and gradually decrease with increasing pressure. It should be noticed here that the experimental points at pressures higher than 150-200 bar are in the one-phase region (see Fig. 1). Then the variation of the molar fractions in this region does not mean change in the solubility of benzene in water but depends on the experimental conditions such as an initial volume ratio of water and benzene and a way to attain the experimental temperature-pressure points, because our cell is not a completely closed system. However, this does not affect the usefulness of the data for studying the volumetric behavior of the mixtures in the following section.

\section{B. Volumetric behavior of the mixtures}

Figure 7 shows the experimental (a) and calculated (b) densities of the water-benzene mixtures. The experimental densities (in $\mathrm{g} \mathrm{cm}^{-3}$ ) are readily given from concentrations of water and benzene as

$$
\rho_{\text {mix }}=\left(C_{W} M_{W}+C_{B} M_{B}\right) / 1000,
$$

where $M_{W}$ and $M_{B}$ are molar weights of water and benzene, respectively. The calculated densities, defined as average densities before mixing, are given by

$$
\rho_{0}=\frac{C_{W} M_{W}+C_{B} M_{B}}{\left(C_{W} M_{W} / \rho_{W}\right)+\left(C_{B} M_{B} / \rho_{B}\right)},
$$

where $\rho_{W}$ and $\rho_{B}$ denote densities of neat water ${ }^{30}$ and neat benzene, ${ }^{29}$ respectively. The discrete jumps in $\rho_{0}$ at 598 and 


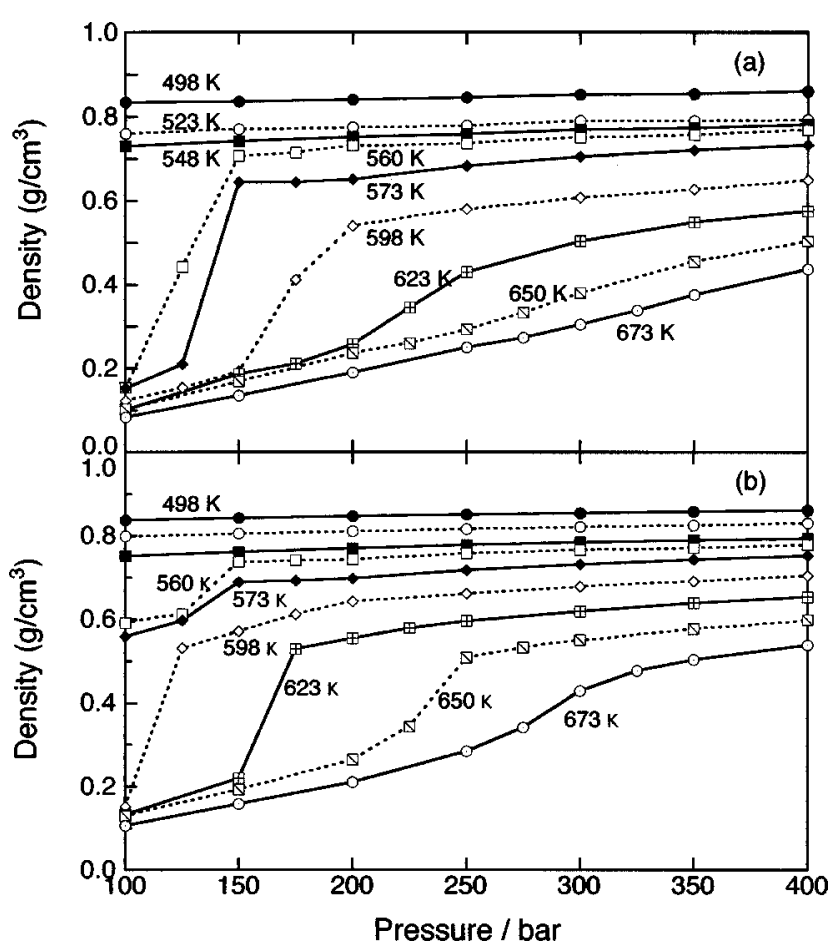

FIG. 7. Experimental densities of the water-rich phase of the water-benzene mixtures (a) and calculated average densities before mixing (b).

$623 \mathrm{~K}$ are caused by the gas-to-liquid transition of neat water. If the molar volumes of the neat components were additive, the experimental densities of the mixtures $\rho_{\text {mix }}$ would agree with the $\rho_{0}$ values. Obviously, this is not the case for the water-benzene mixtures and each of all the experimental densities is smaller than the corresponding calculated density at the same temperature and pressure. This property of the mixtures can be well displayed by the relative volume change on the mixing given by

$$
\frac{\Delta_{\text {mix }} V}{V_{0}}=\frac{V_{\text {mix }}-V_{0}}{V_{0}}=\frac{\rho_{0}}{\rho_{\text {mix }}}-1 .
$$

The resulting values are plotted against pressure at each temperature in Fig. 8. At temperatures below $548 \mathrm{~K}$, the relative volume change is very small, being a few hundredths, and slightly decreases with increasing pressure. This is like an ordinary volumetric behavior of liquid mixtures in the temperature-pressure region far from the critical condition. On the other hand, at the higher temperatures in the twophase region and the one-phase region, the relative volume change becomes anomalously large particularly at low pressures. At $560 \mathrm{~K}$ and $100 \mathrm{bar}$, for example, the relative volume change reaches about 2.8, which means 3.8 times volume expansion on the mixing. The jumps at 598 and $623 \mathrm{~K}$ are due to the jumps in the calculated densities mentioned above. Figure 9 shows a contour map of the relative volume change on the $P-T$ phase diagram, where variations in the relative volume change are expressed by gradation. The darker region indicates higher degree of the volume expansion. It is found that the anomalously large volume expansion occurs in a narrow region, which is enclosed by an extended line of the three-phase equilibrium curve and the one-phase critical curve of the mixtures, and the gas-liquid

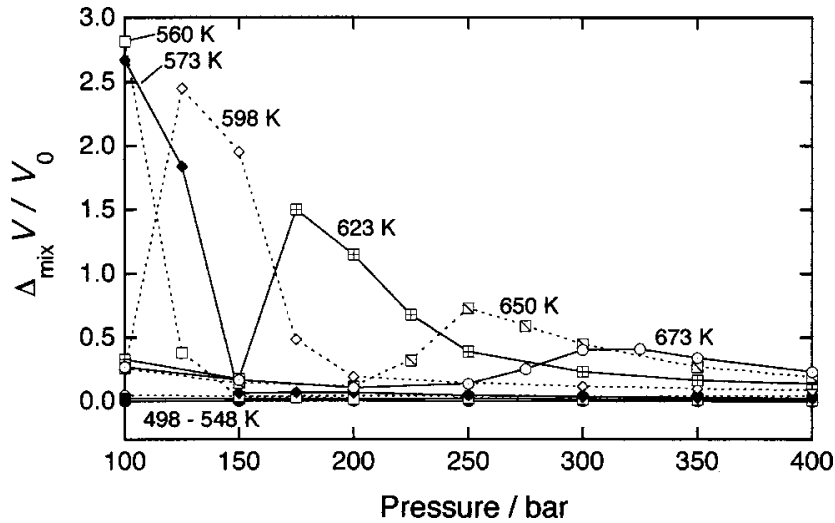

FIG. 8. Relative volume change on the mixing for the water-rich phase of the water-benzene mixtures.

equilibrium curve of water. The volume change is particularly large in the area immediately above the vapor pressure curve of water. This fact suggests that the dissolution of benzene, which itself is in the supercritical condition, induces a density change of the water-rich phase from a liquidlike condition to a gaslike condition. A very similar volumetric behavior to that in Fig. 8 has recently been reported by Furutaka et al. for the hydrocarbon-rich phase of the waterhydrocarbon mixtures. $^{21,22}$ It is proved that the maxima of the volume expansion of both the water-rich and the benzene-rich phases of the mixtures occur at about the same pressure at each temperature of 573, 598, and $623 \mathrm{~K}$. Therefore, the anomalous volumetric behavior should be characteristic of water-hydrocarbon mixtures in the vicinity of the critical region, particularly near the vapor pressure curve of water. This phenomenon may have some relationship with the large excess molar volumes of fluid mixtures of carbon dioxide with ethene and sulfur hexafluoride observed at around room temperature and near the critical pressures of the component fluids. ${ }^{31,32}$

\section{Composition dependence of the relative volume change}

To elucidate a relationship between the volumetric behavior of the water-rich phase and that of the benzene-rich phase, we have examined how the relative volume change varies with the molar fraction of benzene. Near-infrared

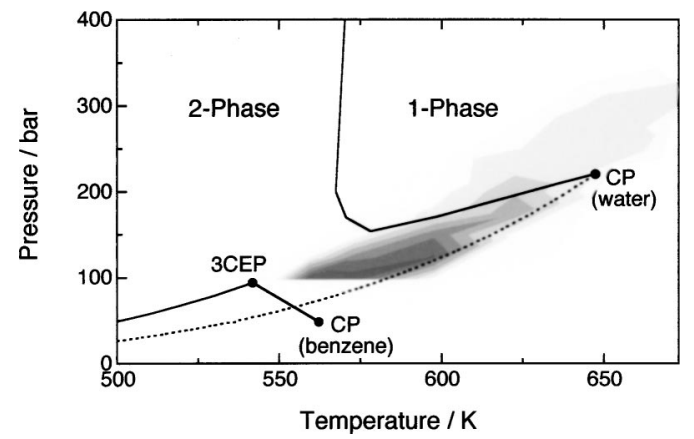

FIG. 9. Contour map of the relative volume change on the pressuretemperature phase diagram of the water-benzene mixtures. The darker region indicates higher degree of the volume expansion. 


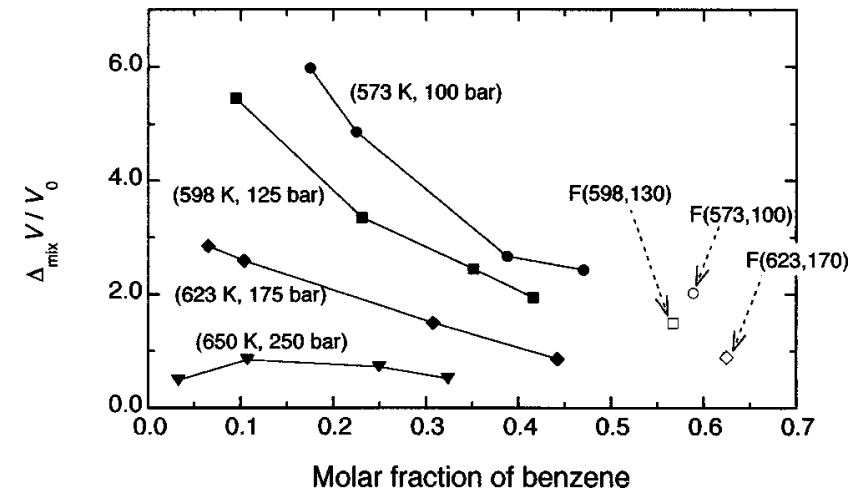

FIG. 10. Plots of the maximum values of the relative volume change against molar fraction of benzene at several temperatures. Previously reported values (Ref. 19) are marked with $F$.

measurements have been performed for samples with different initial volume ratios of water and benzene, which were set at 5.5:0.5, 5.0:1.0, 4.0:2.0, respectively, while the results described so far in this paper are of the sample with the initial ratio of 4.5:1.5. The observed spectra have been treated in the same way as described in the experimental section and the values of the relative volume changes are estimated at various temperatures and pressures. The results have indicated that the pressures at which the maximum volume change occurs at each temperature are the same for all the samples, although the magnitudes of the relative volume change vary with composition. Figure 10 shows plots of the maximum values at a few temperatures against molar fraction of benzene. The previously reported values for the benzene-rich phase $\mathrm{e}^{21}$ are also plotted in Fig. 10 and prove to be consistent with the present results for the water-rich phase. It is found that the magnitude of the volume change on the mixing decreases as the temperature increases from 573 to $650 \mathrm{~K}$ or goes away from the critical temperature of benzene [562.05 K (Ref. 6)]. It is also obvious that the relative volume change decreases with increasing molar fraction of benzene, except at $650 \mathrm{~K}$ and 250 bar where the plots exhibit a little decrease at the lowest benzene fraction. To gain an insight into this fact, it is useful to compare variation patterns of the relative volume changes at different pressures at a constant temperature. Figure 11 displays the relative volume changes at several pressures at $623 \mathrm{~K}$. At pressures except 175 bar, the relative volume change exhibits convexshaped dependence on the molar fraction and seems to converge at zero at zero molar fraction. On the other hand, at $175 \mathrm{bar}$, the relative volume change seems to increase further at the lower molar fraction toward zero. This fact indicates that dissolving a small amount of benzene in liquid water at a specific pressure, which is slightly above the vapor pressure of water [165 bar (Ref. 30) at $623 \mathrm{~K}$ ], induces a transition in the fluid density from liquid to gaslike condition, and the density change becomes milder as the pressure goes up or down from the specific pressure. The corresponding specific pressures at 573 and $598 \mathrm{~K}$ will be near 100 and 125 bar, respectively, as inferred from Fig. 10.

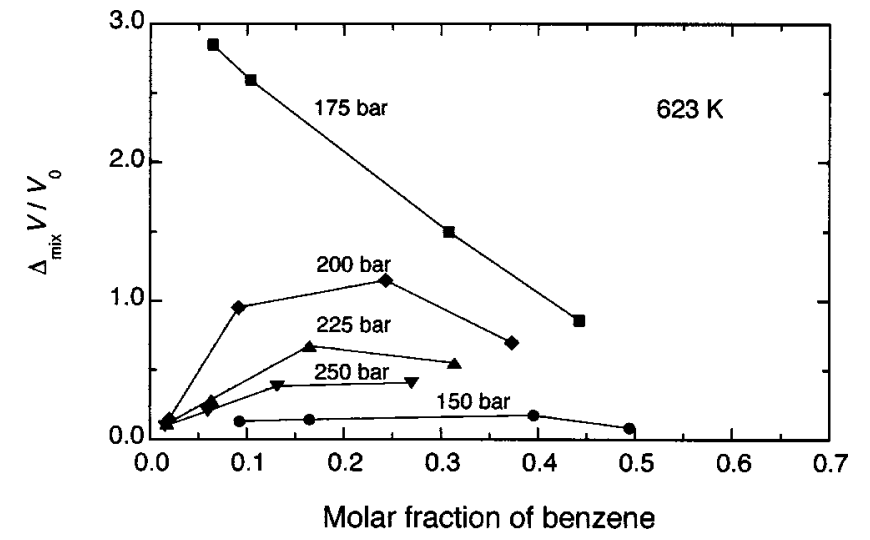

FIG. 11. Plots of the relative volume change of the water-benzene mixtures against molar fraction of benzene at different pressures at $623 \mathrm{~K}$.

\section{ACKNOWLEDGMENTS}

The authors thank Dr. Seiya Furutaka for valuable discussions. They also thank Dr. Takuya Fukuda for his kind support in use of the near-infrared light source. This work was supported by the Grant-in-Aid for Scientific Research from the Ministry of Education, Culture, Sports, Science and Technology of Japan (Grant No. 13440169).

${ }^{1}$ R. J. Rebert and W. B. Kay, AIChE J. 5, 285 (1959).

${ }^{2}$ W. H. Thompson and J. R. Snyder, J. Chem. Eng. Data 9, 516 (1964).

${ }^{3}$ J. F. Connolly, J. Chem. Eng. Data 11, 13 (1966).

${ }^{4}$ Z. Alwani and G. M. Schneider, Ber. Bunsenges. Phys. Chem. 71, 633 (1967).

${ }^{5}$ C. Tsonopoulos and G. M. Wilson, AIChE J. 29, 990 (1983).

${ }^{6}$ CRC Handbook of Chemistry and Physics, 83rd ed. (CRC, Boca Raton, 2002).

${ }^{7}$ J. G. Roof, J. Chem. Eng. Data 15, 301 (1970).

${ }^{8}$ J. S. Rowlinson and F. L. Swinton, Liquids and Liquid Mixtures, 3rd ed. (Butterworth, London, 1982).

${ }^{9}$ E. U. Franck, Ber. Bunsenges. Phys. Chem. 88, 820 (1984).

${ }^{10}$ C. J. Wormald, Ber. Bunsenges. Phys. Chem. 88, 826 (1984).

${ }^{11} \mathrm{~J}$. Li, I. Vanderbeken, S. Ye, H. Carrier, and P. Xans, Fluid Phase Equilib. 131, 107 (1997)

${ }^{12}$ C. J. Wormald, N. M. Lancaster, and C. J. Sowden, J. Chem. Soc., Faraday Trans. 93, 1921 (1997).

${ }^{13}$ I. M. Abdulagatov, A. R. Bazaev, E. A. Bazaev, M. B. Saidakhmedova, and A. E. Ramazanova, J. Chem. Eng. Data 43, 451 (1998).

${ }^{14}$ Th. Hirth and E. U. Franck, Ber. Bunsenges. Phys. Chem. 97, 1091 (1993).

${ }^{15}$ I. Hua, R. H. Hochemer, and M. R. Hoffmann, J. Phys. Chem. 99, 2335 (1995).

${ }^{16}$ T. Sako, T. Sugeta, K. Otake, M. Sato, M. Tsugumi, T. Hiaki, and M. Hongo, J. Chem. Eng. Jpn. 30, 744 (1997).

${ }^{17}$ W. C. Bell, K. S. Booksh, and M. L. Myrick, Anal. Chem. 70, 332 (1998).

${ }^{18}$ Y. Yamasaki, H. Enomoto, N. Yamasaki, and M. Nakahara, Bull. Chem. Soc. Jpn. 73, 2687 (2000).

${ }^{19}$ S. Furutaka and S. Ikawa, J. Chem. Phys. 113, 1942 (2000).

${ }^{20}$ S. Furutaka, H. Kondo, and S. Ikawa, Bull. Chem. Soc. Jpn. 74, 1775 (2001).

${ }^{21}$ S. Furutaka and S. Ikawa, J. Chem. Phys. 117, 1682 (2002).

${ }^{22}$ S. Furutaka and S. Ikawa, Fluid Phase Equilib. 217, 59 (2004).

${ }^{23}$ Y. Jin and S. Ikawa, J. Chem. Phys. 119, 12432 (2003).

${ }^{24}$ E. U. Franck and K. Roth, Discuss. Faraday Soc. 43, 108 (1967).

${ }^{25}$ W. A. P. Luck and W. Ditter, Z. Naturforsch. B 24b, 482 (1969).

${ }^{26}$ G. V. Bondarenko and Yu. E. Gorbaty, Mol. Phys. 74, 639 (1991).

${ }^{27}$ Yu. E. Gorbaty and G. V. Bondarenko, Appl. Spectrosc. 53, 908 (1999).

${ }^{28}$ T. Tassaing, Y. Danten, and M. Besnard, J. Mol. Liq. 101, 149 (2002). 
${ }^{29}$ R. D. Goodwin, J. Phys. Chem. Ref. Data 17, 1541 (1988).

${ }^{30}$ E. W. Lemmon, M. O. McLinden, and D. G. Friend, in NIST Chemistry WebBook, NIST Standard Reference Database Number 69, edited by P. J. Linstrom and W. G. Mallard (National Institute of Standards and Technol- ogy, Gaithersburg, MD, 2001) (http://webbook.nist/gov).

${ }^{31}$ C. J. Wormald and J. M. Eyears, Fluid Phase Equilib. 179, 139 (2001).

${ }^{32}$ C. J. Wormald and R. W. Hodgetts, Fluid Phase Equilib. 187-188, 391 (2001) 